\title{
Recrystallization of Active Pharmaceutical Ingredients
}

\author{
Nicole Stieger and Wilna Liebenberg \\ North-West University, Unit for Drug Research \& Development \\ South Africa
}

\section{Introduction}

Recrystallization can be described simply as a process whereby a crystalline form of a compound may be obtained from other solid-state forms, being themselves crystalline or amorphous, of the same substance. Recrystallization is the process most often employed for the intermediate separation and last-step purification of solid active pharmaceutical ingredients (APIs) (Shekunov \& York, 2000; Tiwary, 2006). Chemical purity is however not the only property of a pharmaceutical active that will affect its performance. Crystal structure (Table 1), crystal habit (Table 2) and particle size all play a part. Polymorphism (different crystal structures of the same substance) affects the physico-chemical properties and stability of an API, whereas crystal habit and particle size mostly affect various indices impacting on dosage form production and performance: particle orientation; flowability; packing and density; surface area; aggregation; compaction; suspension stability; and dissolution (Blagden et al., 2007; Doherty \& York, 1988; Tiwary, 2006).

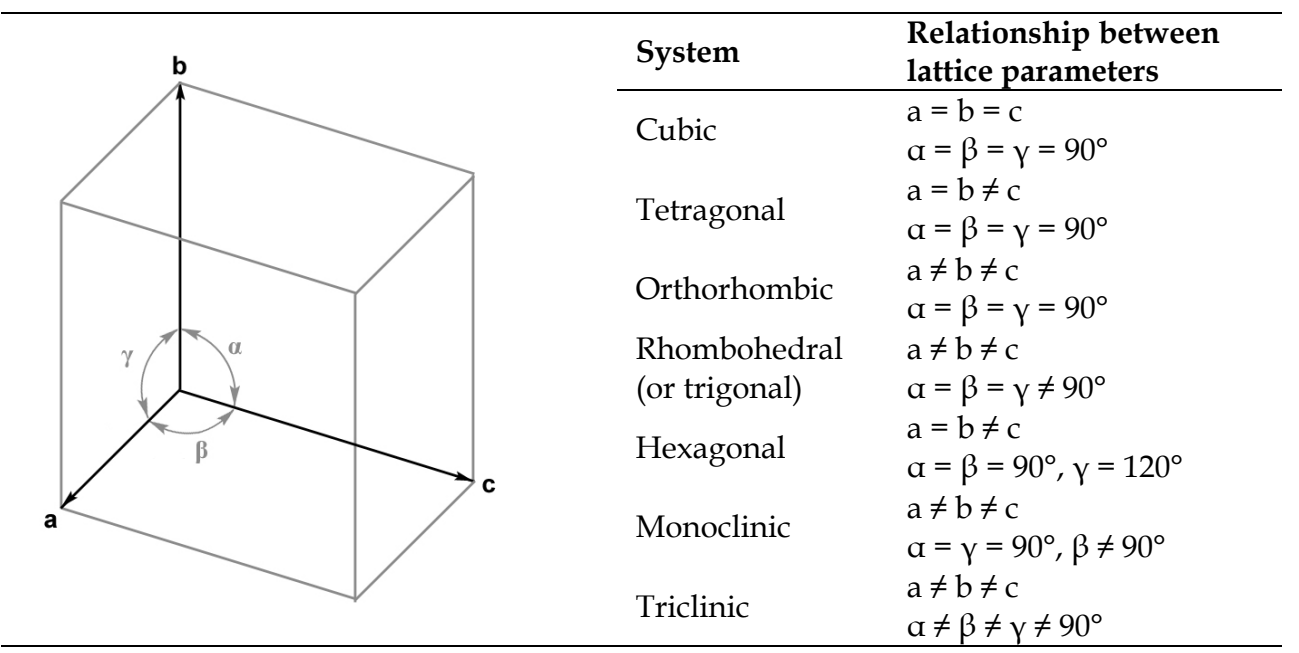

Table 1. Crystal systems and lattice parameters. (Adapted, with permission of Informa Healthcare, from Rodríguez-Homedo et al., 2006.) 


\begin{tabular}{ll}
\hline Crystal morphology & Description \\
\hline Acicular & Slender, needle-like crystal \\
Aggregate & Mass of adhered crystals \\
Blade & Long, thin, flat crystal \\
Dendritic & Tiny crystallites forming a tree-like pattern \\
Equant/cubic & Crystal with similar length, width and thickness \\
Fiber & Long, thin needle; longer than acicular \\
Flake/lath & Thin, flat crystal similar in width and length \\
Plate/platy & Flat crystals with similar width and length but thicker than a flake \\
Prismatic/bipyramid & Hexagonal crystals with faces parallel to the growth axis; width and \\
& thickness greater than acicular and shorter in length \\
Rod & Cylindrical crystals elongated along one axis \\
Rosette/spherulite & Sphere composed of needles or rods radiating from a common center \\
Tablet/tabular & Flat crystal with similar width and length but thicker than a plate \\
\hline
\end{tabular}

Table 2. Descriptions of common pharmaceutical crystal morphologies. (Adapted, with permission of Informa Healthcare, from Rodríguez-Homedo et al., 2006.)

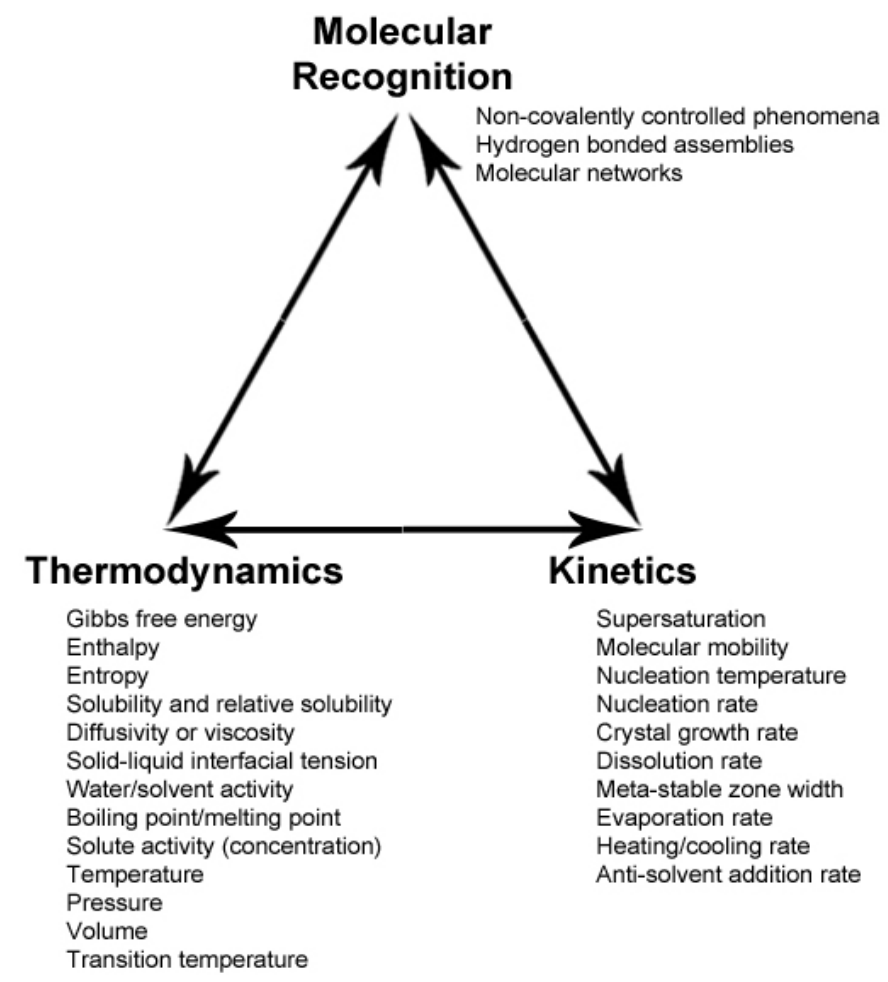

Fig. 1. Diagram showing the phenomena that govern solid-state transformations.

Mechanical, thermal and chemical (solvents, additives, impurities, relative humidity) stresses affect the competition (or reinforcement) among these processes. (Adapted, with permission of Informa Healthcare, from Rodríguez-Homedo et al., 2006.) 
The number of solid-state forms that an API could exist in relies on the range of noncovalent interactions and molecular assemblies, the order range, and the balance between entropy and enthalpy that defines the free energy states and processes (Figure 1). When an API exists as more than one solid-state form, thermodynamics control their relative stability and the conditions and direction in which a transformation can occur, whilst kinetics determine how long it will take for a transformation to reach equilibrium. Thermodynamics establish the stability domains of the solid-states, but once a metastable domain is encountered, the kinetic pathways will determine which form will be created and for how long it will survive (Rodríguez-Spong et al., 2004).

Small changes in recrystallization procedure can influence the crystallization process and may lead to changes in API crystal structure, crystal habit and particle size with subsequent variability of raw material characteristics and dosage form performance. Herein lies the challenge and the opportunity: manufacturers of pharmaceutical actives go to great lengths to ensure production uniformity, but a pharmaceutical researcher might choose to alter recrystallization conditions to manipulate API characteristics.

Crystallization is generally thought of as the evolution from solution or melt of the crystalline state (Blagden et al., 2007), but a broader definition includes precipitation and solid-state transitions (Shekunov \& York, 2000). Crystallization methods can be solvent or non-solvent based and the varied reaction conditions generate different crystal forms (Banga et al., 2004). Non-solvent based methods include sublimation, thermal treatment, desolvation of solvates, grinding and crystallization of a melt (Guillory, 1999). Applied in polymorph screening studies, traditional crystallization approaches most often will not yield all possible polymorphs of a given API. Therefore, there is a continuous search for innovative methods of manipulating the crystallization process (Rouhi, 2003).

\section{Solvent based recrystallization}

In solution, crystallization is the creation of a crystalline phase by a process (Figure 2) initiated by molecular aggregation, leading to the formation of nuclei (the smallest possible units with defined crystal lattice) and ultimately crystal growth (Banga et al., 2004).

\subsection{Parameters that influence nucleation and crystal growth}

The sections that follow will address factors that influence solvent recrystallization in general and will not venture into the specifics of specialized solvent-based production methods like spray-drying, freeze-drying, etc. The reader should keep in mind that although different parameters are discussed under separate headings, they are interactive and not independent of each other. For example: changing the composition of a solvent system will change the level of saturation; changes in temperature alter viscosity and level of saturation; agitation may increase temperature; etc.

Each of the parameters discussed can influence polymorphism, and polymorphism in turn affects crystal habit. It is also well-known that each polymorph of an API can exhibit multiple crystal habits, therefore a parameter might influence crystal habit without changing the internal structure of the crystals produced (Tiwary, 2006). 


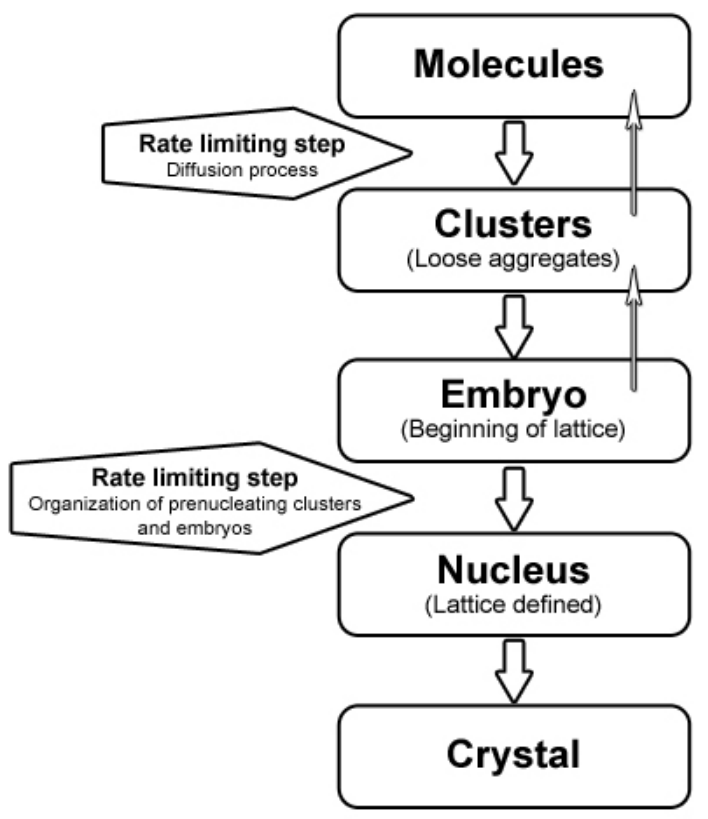

Fig. 2. The course of crystallization and its rate-limiting steps. (Adapted, with permission of Touch Briefings, from Banga et al., 2004.)

\subsubsection{Concentration and temperature}

The difference in chemical potential between the crystallization solution and the solid phase is the fundamental driving force for crystallization, but it is more convenient to express the driving force in terms of supersaturation (Fujiwara et al., 2005). Supersaturation (the difference between solution concentration and saturation concentration at a specific temperature) leads to the creation of metastable (far from equilibrium) liquid states and crystallization provides a means to reduce the free energy of the system to the most stable state (equilibrium) (Rodríguez-Homedo et al., 2006). The kinetics of nucleation and crystal growth are strongly dependent on supersaturation (Braatz, 2002; Togkalidou et al., 2002). An increase in the degree of supersaturation of a solution leads to a reduction in the size of crystals produced. At high supersaturation nucleation is more rapid than growth, resulting in the precipitation of fine particles (Carstensen et al., 1993; Tiwary, 2006). It is therefore important to control the degree of supersaturation during crystallization, because the size, shape and solid-state form of the crystals produced are all influenced by supersaturation (Fujiwara et al., 2005).

Supersaturation is typically achieved through processes that either increase the solute concentration (evaporation or dissolution of a metastable solid phase with subsequent transformation to the more stable, but less soluble form) or decrease the solubility of the solute (cooling, addition of an antisolvent, $\mathrm{pH}$ change or the addition of ions that participate in precipitation of the solute) or through a combination of these strategies (Fujiwara et al., 2005; Rodríguez-Homedo et al., 2006). 
The main effect of temperature on crystallization from solution is secondary to its influence on the solubility of the solute, and subsequently the degree of saturation of the solution. Although molecular recognition is required for the formation of molecular clusters, and this is dependent on molecular mobility and collision rates, both of which increase at higher temperatures, the molecular mobility in liquids is too high to be the rate-limiting factor in nucleation and crystal growth (Rodríguez-Spong et al., 2004).

The relationship between nucleation rate and supersaturation is well known and Figure 3 illustrates that the number of nuclei generated by a high rate of cooling increases exponentially with increasing supersaturation. A high number of nuclei at the outset limits their growth potential (Tung et al., 2009).

In cases where the crystallization of a metastable polymorph precedes in-solvent transformation to a more stable polymorph as described by Ostwald's Rule of Stages (Boistelle \& Astier, 1988; Ostwald, 1897), the solvent-mediated transformation can be affected by the temperature of the crystallization medium (Stieger et al., 2009), because the process is thermally activated (Beckman, 2000).

Temperature cycling or oscillation is sometimes used to accelerate the effect of postcrystallization Ostwald Ripening in slurries containing just one polymorph of an API. Small particles and rough edges of larger particles dissolve faster during heating periods, followed by their recrystallization onto the existing crystals during cooling. The overall effect is more uniformly shaped particles and a narrower particle size distribution range (Kim et al., 2003; Mullin, 2001).

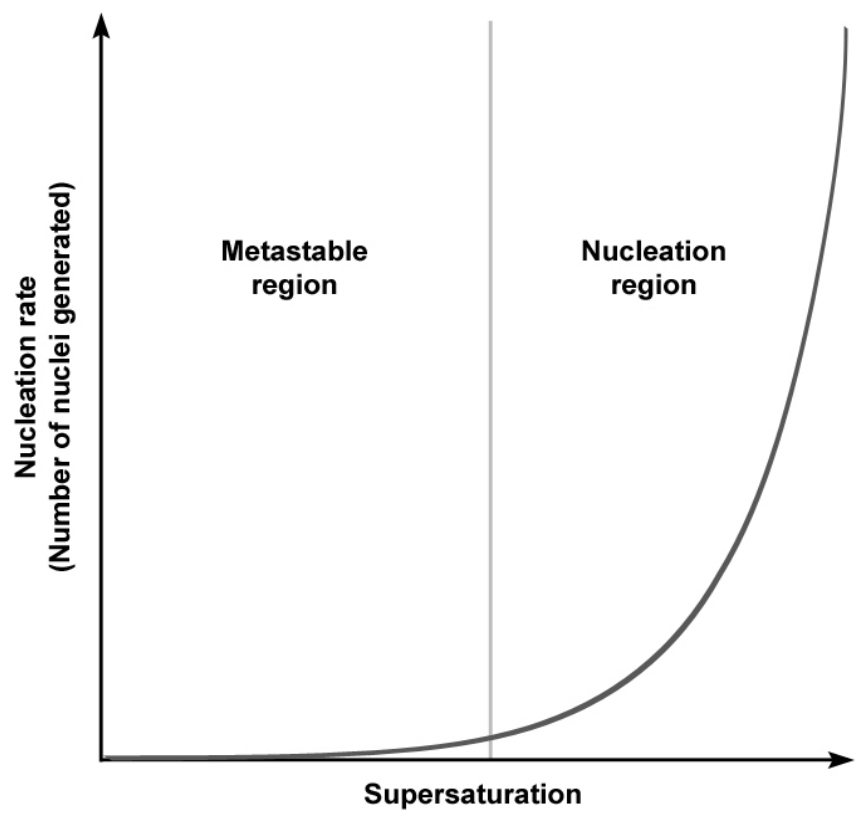

Fig. 3. Nucleation versus supersaturation (Adapted, with permission of John Wiley and Sons, from Tung et al., 2009). 


\subsubsection{Interfaces and surfaces}

Nucleation can be either homogeneous or surface catalyzed (Figure 4). Homogeneous nucleation seldom occurs in volumes greater than $100 \mu \mathrm{l}$, because solutions contain random impurities that may induce nucleation. Surface catalyzed nucleation can be promoted by surfaces of the crystallizing solute (secondary nucleation) or a surface/interface of different composition than the solute may induce nucleation (heterogeneous nucleation) by decreasing the energy barrier for the formation of nuclei (Rodríguez-Homedo et al., 2006).

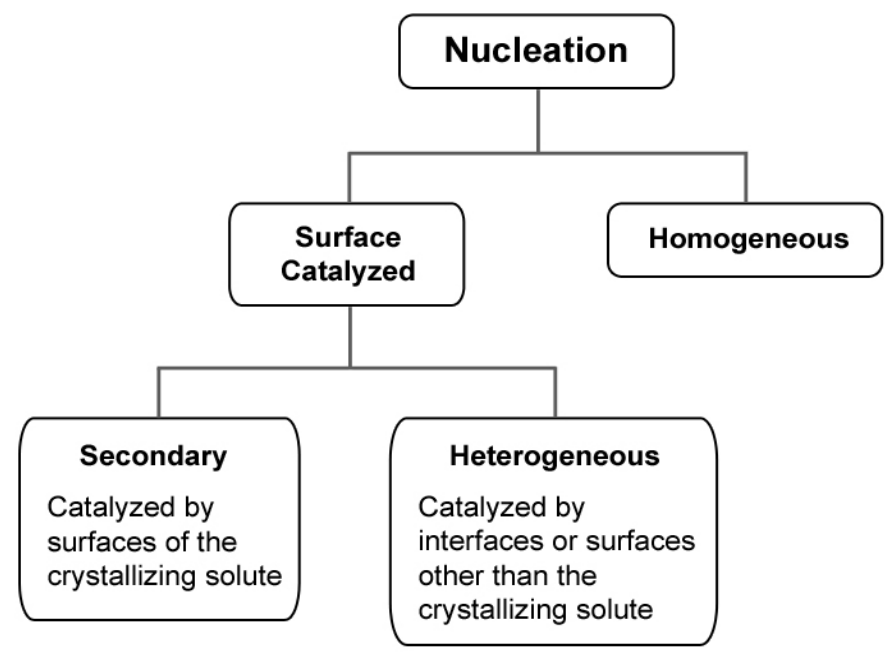

Fig. 4. Mechanisms for crystal nucleation. (Adapted, with permission of Informa Healthcare, from Rodríguez-Homedo et al., 2006.)

Surfaces promoting heterogeneous nucleation may be introduced into the crystallization solution intentionally (as a means of controlling crystal form of the product) (RodríguezSpong et al., 2004) or unintentionally (dust and other impurities), or they may be an unavoidable part of the process (crystallization vessel, vessel-solution interface and the solution-air interface) (Florence \& Attwood, 2006; Kuzmenko et al., 2001).

The intentional introduction, into crystallization solutions, of surfaces that catalyze nucleation is known as "seeding". Usually, seeding is performed by introducing crystals of the solute that have the preferred crystal structure one wishes to obtain. Seeding can also be performed using isomorphous substances that differ from the solute (Florence \& Attwood, 2006). Seeding techniques can be applied to initiate crystallization; to control particle size usually when larger crystals with uniform size distribution are required; to avoid encrustation through spontaneous nucleation; to control polymorphic form; and to obtain crystals of high purity, high perfection, desired orientation and sufficient size for crystal structure determination by X-ray diffraction (Beckman, 2000).

When the concentration of an API in the crystallization medium is increased past its solubility curve (Figure 5), whether by cooling or by evaporation of solvent, nucleation does not immediately occur. The solution has to reach a certain concentration-temperature point 
where spontaneous nucleation occurs - this is the border of the metastable zone. For seeding to be successful in producing just the required product, the concentration and temperature of the crystallization medium must be strictly controlled within this zone. If the solubility line is crossed to the left, the seed crystals will dissolve and if the metastable zone limit is crossed to the right, spontaneous nucleation will take place and could result in unwanted crystal forms (Beckman, 2000; Fujiwara et al., 2005).

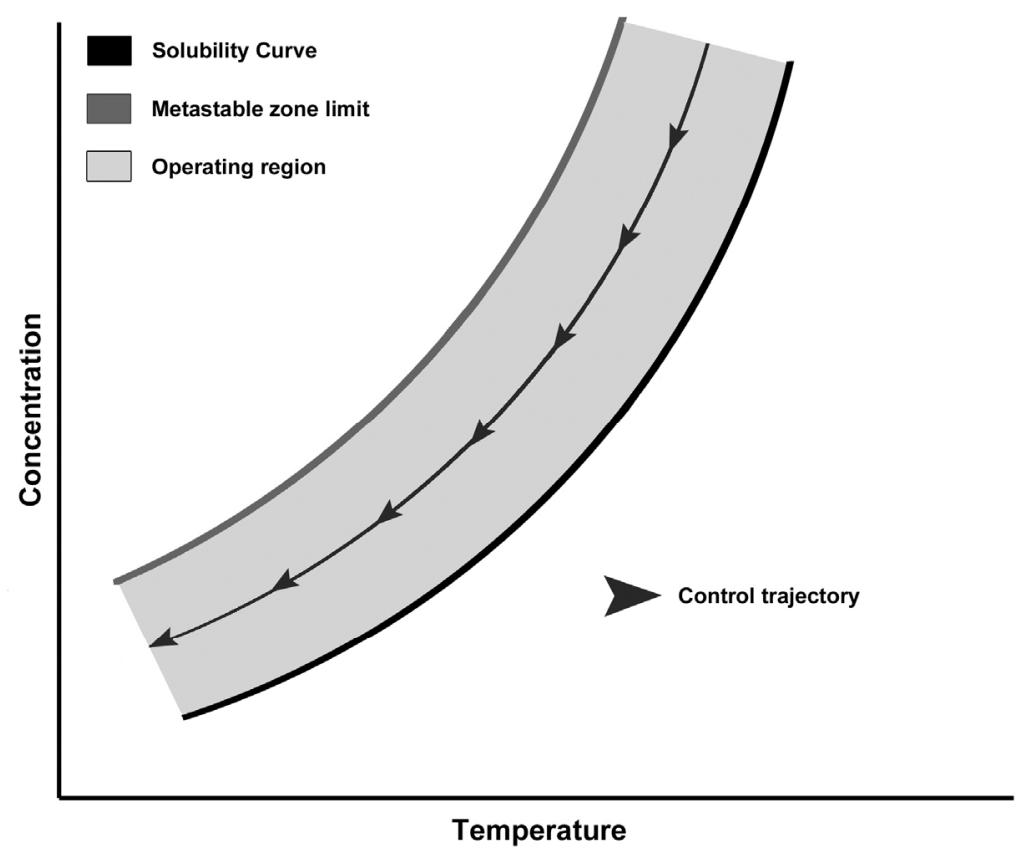

Fig. 5. The operating region of seeded industrial batch crystallization is the metastable zone, which is bound by the solubility curve and the metastable limit for the specific API. The concentration-temperature profile (control trajectory) to be used lies within the metastable zone (operating region). (Adapted, with permission of Elsevier, from Fujiwara et al., 2005.)

In evaporative crystallization, crystals are sometimes observed to form preferentially near the surface of the solution, due to a higher local concentration of solute. The meniscus of a solution can also have geometry favoring higher evaporation rates, with crystals then forming at the contact line with the crystallization vessel (Capes \& Cameron, 2007).

\subsubsection{Solvent}

Dependent on the conditions employed, the crystallization of API polymorphs from a solvent may be under kinetic or thermodynamic control. When crystallization of a dimorphic compound (Figure 6) is conducted sufficiently above or below the transition point, in an area defined by the solubility curves of the two polymorphs, the solvent used is immaterial provided that the API solubility is adequate to allow the prescribed concentrations to be reached. Irrespective of the kinetics, the outcome is under total 
thermodynamic control. If crystallization takes place outside the area described above (B2, C1, D1, E1 and E2), the choice of solvent may or may not be critical. This will be determined by the relative kinetics of formation, growth, and transformation of the two polymorphs in the various solvents (Threlfall, 2000).

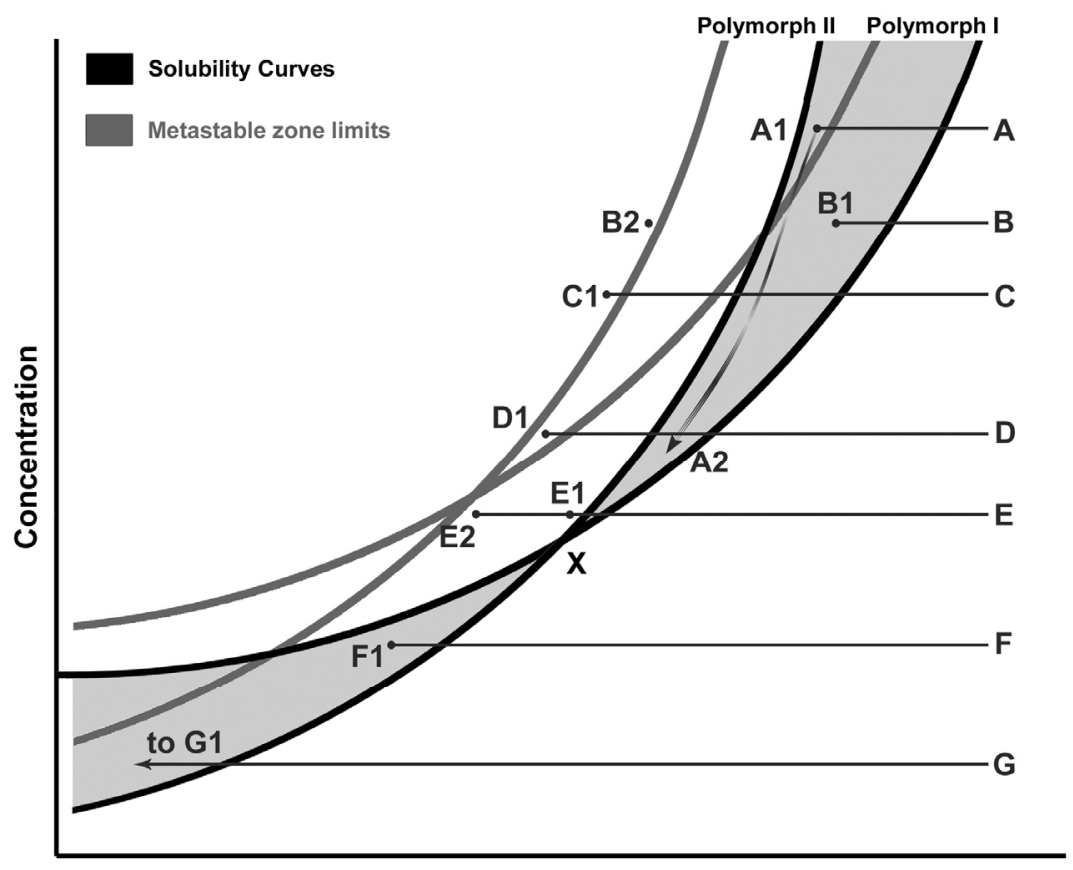

Temperature

Fig. 6. Polymorphic system of two enantiomorphically related polymorphs I and II. (Transition point $X$ at $T_{X}$; A-G, initial state of hot, undersaturated solutions; A1-G1, B2 and $\mathrm{E} 2$, state of solution at point of initial crystallization. If $\mathrm{B}$ is seeded at B1, it behaves as A1.) (Adapted, with permission of the American Chemical Society, from Threlfall, 2000.)

Recrystallization from solvents often leads to the isolation of solvates, in fact, API solvates are very common (Griesser, 2006). Although they have a recognized potential to improve dissolution kinetics (Brittain \& Grant, 1999; Haleblian, 1975; Tros de Ilarduya et al., 1997), they are rarely selected for further development or dosage form formulation - the only exceptions being hydrates. The main reasons for the rarity of marketed API solvates are their solid-state metastability and the relative toxicity of any included solvent (Douillet et al., 2011). It goes without saying that, in the production of solvates, the solvent/s used for recrystallization will be the one/s that could potentially be included in the crystal lattice.

The nature of the crystallization solvent can affect crystal habit, regardless of change in polymorphism (Stieger et al., 2010a). The interaction of the solvent at the different crystalsolution interfaces may lead to altered roundness of growing crystal faces and/or edges, changes in crystal growth kinetics, and enhancement or inhibition of crystal growth at certain faces (Figure 7), thereby changing the crystal habit (Stoica et al., 2004; Tiwary, 2006). 


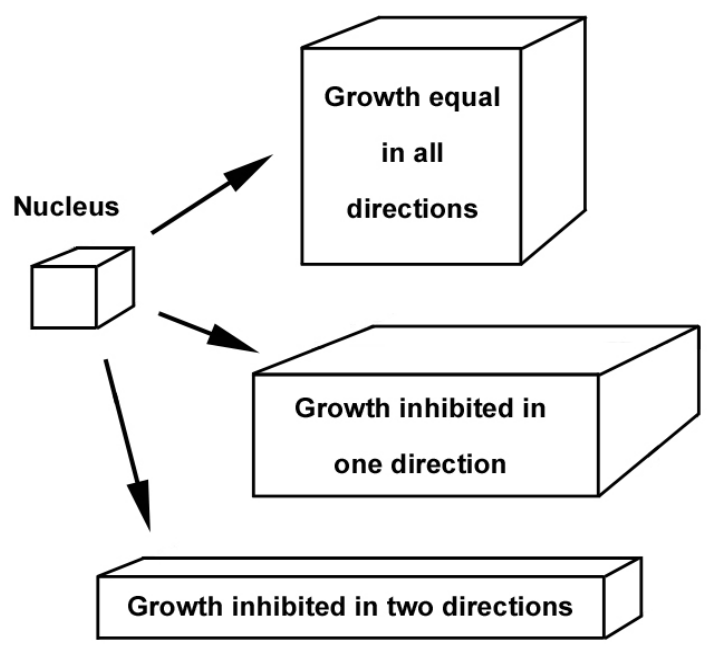

Fig. 7. Crystal habits arising from growth inhibition at crystal faces. (Adapted, with permission of Informa Healthcare, from Carstensen et al., 1993.)

\subsubsection{Agitation, mixing and stirring}

Mixing of crystallization solutions and crystal slurries is often necessary, especially in industry, to ensure homogeneity (heat transfer, dispersion of additives, uniformity of crystal suspension, avoidance of settling, etc.). Its effects on nucleation, both primary and secondary, and crystal growth are far reaching and complex. An unagitated solution can, in general, be cooled further before onset of nucleation, because the overall result of mixing is a decrease in the width of the metastable zone. In a mixed solution at constant supersaturation, with no crystals yet present, mixing intensity can reduce induction time the time elapsed before crystals first appear. Induction time decreases up to a critical speed and then remains unchanged (Karpinski \& Wey, 2001; Mullin, 2001; Tung et al., 2009).

Mixing actively generates secondary nuclei through crystal-crystal, crystal-vessel and crystal-impeller impacts. A greater number of nuclei generated in agitated systems leads to a decrease in the ultimate crystal size of the product. Mixing also has an intensity dependent effect on the mass transfer rate of growing crystals with a growth limiting outcome (Tung et al., 2009).

It has been found that stirring can reproducibly affect the chiral symmetry of crystallization products. If a substance crystallizes as an equal mixture of dextro and levo crystals when unstirred, its chiral symmetry can be disrupted by stirring (Kondepudi et al., 1990; McBride \& Carter, 1991). This phenomenon has been attributed to the effect of stirring on secondary nucleation (Kondepuddi \& Sabanayagam, 1994).

\subsubsection{Pressure}

Almost all polymorph screening studies and industrial crystallization processes are performed under ambient pressure, but low pressure is also often applied to increase the 
rate of solvent evaporation. As with rapid cooling, rapid evaporation leads to higher nucleation rates.

Only recently have researchers turned their attention to high pressure recrystallization from solution as an added dimension in the search for new pharmaceutical polymorphs and solvates. High pressure encourages denser structures in which molecules must pack more efficiently and this means that changes in relative orientations are likely to occur. This gives rise to different themes of molecular interactions, the strengths of which are in turn sensitive to distance and therefore the effects of pressure. The interactions between solute and solvent molecules are also modified by pressure, changing the solubility of a given polymorph or solvate. In some instances, the differences in solubility between two forms might also change, thereby encouraging recrystallization of one form at the expense of another (Fabbiani et al., 2004).

\subsubsection{Moisture and humidity}

When recrystallizing from hygroscopic organic solvents, care should be taken to use only properly dried solvents (Table 3 ) or newly opened containers from reputable suppliers. If a crystallization process generates a metastable intermediary form, it can absorb water when exposed to moisture and change into a hydrate. Water molecules, because of their small size and multidirectional hydrogen bonding capabilities, are particularly suited to fill structural voids (Manek \& Kolling, 2004). If a crystallization system is open to atmospheric conditions, as is likely to be the case with evaporative crystallization, hygroscopic solvents will absorb moisture from the air if present. Should an evaporative crystallization process be sensitive to the presence of moisture, additional measures will have to be put in place to eliminate atmospheric humidity.

\begin{tabular}{ll}
\hline Organic Solvent & Drying Agent \\
\hline Alcohols & $\begin{array}{l}\text { Anhydrous potassium carbonate; anhydrous } \\
\text { magnesium or calcium sulphate; calcium oxide. } \\
\text { Anhydrous calcium chloride; anhydrous sodium, } \\
\text { magnesium or calcium sulphate; phosphorous } \\
\text { Aryl halides }\end{array}$ \\
pentoxide. \\
hydrocarbons & Anhydrous calcium chloride; anhydrous calcium \\
Ethers & sulphate; metallic sodium; phosphorous pentoxide. \\
Aldehydes & Anhydrous sodium, magnesium or calcium sulphate. \\
Ketones & Anhydrous sodium, magnesium or calcium sulphate; \\
& anhydrous potassium carbonate. \\
Organic bases (amines) & Solid potassium or sodium hydroxide; calcium oxide; \\
Organic acids & arium oxide. \\
Halogenated solvents* & Anhydrous sodium, magnesium or calcium sulphate. \\
\hline
\end{tabular}

* Never dry halogenated solvents with alkali metals or alkali metal hydrides as this can cause violent explosions.

Table 3. Common drying agents for organic solvents. (Adapted, with permission of Indian Streams Research Journal, from Shinde et al., 2011.) 


\subsubsection{Addition of salts, polymers or antisolvents}

The addition of salts, polymers or antisolvents can be used to create supersaturation of the solution by decreasing the solute solubility in the crystallization medium. Ions, polymeric molecules, or other substances introduced into the crystallization solvent can also act as impurities for growing crystals. These substances may get adsorbed in the crystal lattice of a growing crystal and disturb the regular and repeating arrangements of the crystal, creating defects and leading to polymorphic modifications. Impurities, and surfactants in particular, can also inhibit crystal growth at certain crystal faces, resulting in crystal habit changes (Tiwary, 2006).

When selecting suitable solvents for antisolvent crystallization, one should select a pair that is miscible. The solute must be more soluble in one solvent and less soluble in the other (antisolvent). A water-soluble API will most likely be more soluble in a polar solvent and less soluble in a non-polar solvent (Table 4). The opposite holds true for a poorly watersoluble API. Antisolvent crystallization is performed by dissolving the API in the solvent and then gradually adding an antisolvent. This results in a decrease of solute solubility and an increase in supersaturation - much like cooling crystallization (Nonoyama et al., 2006; Stieger et al., 2010a; Zhou et al., 2006). Reverse addition is a variation of antisolvent recrystallization whereby the API solution is added to the antisolvent. The resulting rapid increase in supersaturation leads to swift nucleation and the precipitation of very fine particles (Tung et al., 2009). The composition of the crystallization medium can affect both the crystal form and crystal habit of the product (Stieger et al., 2010a).

\begin{tabular}{cccc}
\hline \multicolumn{2}{c}{ Solvent 1 (more polar) } & \multicolumn{2}{c}{ Solvent 2 (less polar) } \\
\hline Solvent & Dielectric constant $(\boldsymbol{\varepsilon})$ & Solvent & $\begin{array}{c}\text { Dielectric constant } \\
(\boldsymbol{\varepsilon})\end{array}$ \\
\hline Water & 78.3 & Ethanol & 24.3 \\
Water & 78.3 & Acetone & 20.7 \\
Methanol & 32.6 & Dichloromethane & 9.08 \\
Ethanol & 24.3 & Acetone & 20.7 \\
Acetone & 20.7 & Diethyl ether & 4.34 \\
Acetone & 20.7 & Petroleum ether & 1.90 \\
Diethyl ether & 4.34 & Hexane & 1.89 \\
Ethyl acetate & 6.02 & Cyclohexane & 1.97 \\
Ethyl acetate & 6.02 & Petroleum ether & 1.90 \\
Dichloromethane & 9.08 & Petroleum ether & 1.90 \\
Toluene & 2.38 & Petroleum ether & 1.90 \\
\hline
\end{tabular}

Table 4. Common solvent-antisolvent pairs. (Adapted, with permission of the author, from Skonieczny, 2009.)

\subsection{Novel strategies and new trends in solvent-based crystallization}

One of the biggest challenges for pharmaceutical researchers is finding all the solid-state forms of an API that can exist at ambient conditions. There is no single method for producing all conceivable forms and a particular polymorph may go undetected for many years. Scientists are continually searching for novel ways of uncovering hidden solid-states. A few of these methods are briefly discussed below. 


\subsubsection{Laser-induced crystallization}

Non-photochemical laser-induced nucleation (NPLIN) is a crystallization technique that can affect both nucleation rate and the crystal form produced. Laser pulses act predominantly on pre-existing molecular clusters by assisting in the organization of pre-nucleating clusters and embryos into nuclei (Figure 2), leading to dramatically increased nucleation rates for supersaturated solutions. It is believed that the plane-polarized light aligns the prenucleating clusters and thereby reduces the entropic barrier to the free energy of activation for critical nucleus formation (Banga et al., 2004; Garetz et al., 1996; Rodríguez-Spong et al., 2004; Zaccaro et al., 2001). This method has not yet been applied to pharmaceuticals, but it should produce similar results as for other organic substances.

\subsubsection{Capillary crystallization}

In order to access metastable forms of an API, high levels of supersaturation are often required. Capillary tubes as recrystallization vessels are ideal for manipulating the metastable zone width through slow evaporation and because the small volumes of solution isolate heterogeneous nucleants, and reduce turbulence and convection. This technique offers the additional advantage that the crystals need not be removed from the capillary tube prior to characterization by single crystal- or powder X-ray diffraction (Banga et al., 2004; Rodríguez-Spong et al., 2004).

\subsubsection{Sonocrystallization}

This technique utilizes ultrasound to increase nucleation rate, but it is also an effective means of crystal size reduction that eliminates many of the disadvantages associated with mechanical size reduction. Sonic waves give rise to a phenomenon called cavitation - the formation of bubbles that decrease in size until a critical size is reached, leading to collapse and the formation of cavities. Cavitation provides energy that accelerates the nucleation process (Banga et al., 2004; Kim et al, 2003).

\section{Non-solvent based recrystallization}

Much emphasis has been placed on the importance of solvent based recrystallization in the production of different solid-state forms of APIs, but non-solvent based recrystallization is equally important to industry and researchers alike. Recrystallization through solid-state transitions affects not only the production of APIs, but also the stability of the final product. Additionally, physical vapor deposition (PVD) recrystallization offers yet more opportunities for the preparation of polymorphs.

\subsection{Recrystallization through solid-state transitions}

Polymorphs may be obtained through solid-state transition by recrystallization of metastable polymorphs and amorphous forms, or through desolvation of solvates (including hydrates). No solid-state transition will take place unless it is thermodynamically favored and solids will always tend to transform to their lowest energy state - the most stable form. Stability is of major concern where APIs are concerned, but it is also true that stable forms are less soluble than their metastable counterparts and solubility of an API 
often affects its bioavailability, and therefore also its efficacy (Lipinsky et al., 1997). This necessitates a compromise between stability and solubility: we search for a metastable form with improved solubility that is still relatively stable enough to withstand the rigors of pharmaceutical processing and will yield a product with acceptable shelf-life.

\subsubsection{Transformation of polymorphs}

Polymorphism, occurring in a single-component crystalline molecular solid, may display monotropism or enantiotropism or both (Figure 8). In a monotropic system (A and C, B and $\mathrm{C})$, only one polymorph is stable below the melting point and a phase change from the metastable form (A and B) to the stable form (C) is irreversible. For enantiotropic systems (A and $B)$, a reversible phase transition is observed at a definite transition temperature $\left(T_{t, A-B}\right)$, where the free energy curves intersect before the melting point $\left(T_{m}\right)$. At temperatures and pressures below $T_{t, A-B}$ form A will be stable (lower free energy and solubility), whilst in the temperature and pressure range between $T_{t, A-B}$ and $T_{m, B}$ form $B$ is more stable. Polymorphs in an enantiotropic system are referred to as enantiotropes and those in a monotropic system are monotropes (Bernstein 2002; Grant 1999).

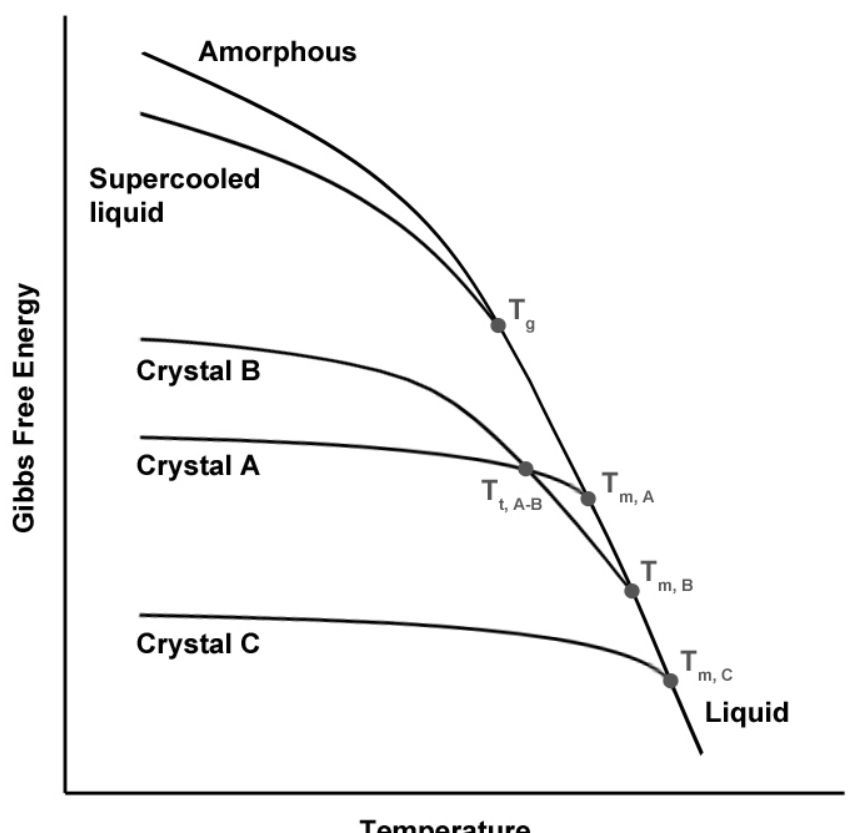

Fig. 8. Gibbs free energy curves for a hypothetical single-component system that exhibits crystalline and amorphous phase transitions. Monotropic systems (A and C, B and C), enantiotropic system (A and $B$ ) with a transition temperature $T_{t}$, and an amorphous and super-cooled liquid with a glass transition temperature $T_{\mathrm{g}}$. Melting points, $\mathrm{T}_{\mathrm{m}}$, for the crystalline phases are shown at the intersection of curves for the crystalline and liquid states. (Adapted, with permission of the authors, from Shalaev \& Zografi, 2002.) 


\subsubsection{Effects of pharmaceutical processing}

Unintentional solid-state conversion of polymorphs sometimes occurs upon exposure to the energetics of pharmaceutical processing and a variety of phase conversions are possible when APIs are exposed to milling, wet granulation, oven drying, and compaction (Brittain \& Fiese, 1999):

Grinding or milling is often the last step in the production of bulk APIs and it is performed to reduce particle size and improve particle size homogeneity. Milling can impart a significant amount of energy on a solid and could potentially lead to a full or partial polymorphic conversion or generation of an amorphous substance (or at least a degree of amorphous content). Amorphous forms, being metastable, can in turn reconvert to a crystalline state which may differ from the original product.

Wet granulation, used to improve powder flow and blend homogeneity, is a step that often precedes the production of tablets. During this process, the API is exposed to a solvent (water or an organic solvent with low toxicity, like ethanol) and is once more prone to undergoing solvent-mediated transformations. Exposure to humidity can create similar conditions which could lead to a hydrate being formed.

Drying of APIs is typically achieved with heat and moving air. The possible ramifications of temperature change on polymorph stability have been discussed in the previous section. Drying conditions need to be carefully controlled to avoid possible transformations.

Although not generally a common occurrence, compaction (during tablet manufacturing) can potentially cause metastable polymorphs to convert to the stable form. This can be attributed to the energy applied to bring about compaction. The extent of transformation is dependent on the zone of the tablet, the pressure applied, the compression temperature and the particle size of the API powder.

\subsubsection{High-pressure polymorphic transitions}

Solid-state pressure-induced structural changes in molecular crystals can cause polymorphic transitions (Boldyreva, 2003). However, unlike with high-pressure solvent recrystallization (please refer to section 2.1.5) which is experimentally and mechanistically similar, the conversion tends to be only partial and the data are poor. It is thought that even though the application of high pressure to larger organic molecules may thermodynamically favor the adoption of a new polymorphic form, the solid-state has a substantial kinetic barrier to overcome before the molecules are mobile enough to rearrange (Fabbiani et al., 2004).

\subsubsection{Desolvation of hydrates and solvates}

With the exception of hydrates that are often used in pharmaceutical dosage forms, most solvates are the penultimate solid form in the production of APIs (Byrn et al., 1999). Solvates may be desolvated by removing the recrystallization solvent and exposing the crystals to air at ambient temperature, leading to a decrease in vapor pressure of the solvent. If a solvate is stable under these conditions, it may be dried under vacuum or in an oven at mild temperatures. 
Desolvation of a solvate generally results in one of the following forms (Byrn et al., 1999):

- An unsolvated polymorph with a crystal structure different to that of the solvate.

- An unsolvated polymorph with a crystal structure that is the same as that of the solvate.

- An amorphous material that may or may not recrystallize.

Sometimes, a polymorphic transition may mimic the first of these scenarios. Nevirapine's metastable Form IV is isostructural to a series of solvates prepared from primary alcohols (Stieger et al., 2010b) except that its structure contains no solvent molecules. When removed from its recrystallization medium, Form IV will spontaneously and rapidly convert to the stable Form I in exactly the same way as the aforementioned solvates (Stieger et al., 2009).

\subsubsection{Crystallization from amorphous phases}

Amorphous pharmaceutical solids may be prepared by common pharmaceutical processes including melt quenching, freeze- and spray-drying, milling, wet granulation and desolvation of solvates (Yu, 2001). Glasses are most often produced from a melt of an API. If crystallization does not occur on cooling the melt below the melting point $\left(\mathrm{T}_{\mathrm{m}}\right)$ of the crystalline phase, a supercooled liquid is obtained. Further cooling induces a change to a glassy state at the glass transition temperature $\left(\mathrm{T}_{\mathrm{g}}\right)$, accompanied by a dramatic decrease in molecular mobility and heat capacity. In contrast to melting point, the glass transition temperature of a compound can fluctuate with operating conditions (including the rate of cooling/heating) and as a function of the history of the sample. This indicates that the glass transition is a thermal event affected by kinetic factors (Petit \& Coquerel, 2006).

An amorphous or glass form is often an intermediate in the production of crystalline APIs and such processes can be useful for overcoming specific kinds of activation energies. Thermodynamically, amorphous solids are out-of-equilibrium states that contain an excess of stored energy, with reference to the crystalline phases (Figure 9), making them unstable by definition. The excess energy can be released either completely through crystallization or partially by means of irreversible relaxation processes (Petit \& Coquerel, 2006).

Crystallization from amorphous solids, as for any crystallization, involves successive nucleation and growth steps. The crystallization rate may be affected by numerous, possibly interdependent, parameters notably including temperature and plasticizers (of which water is one). When the temperature of a supercooled liquid decreases from the melting point to the glass transition temperature, the nucleation rate increases exponentially whereas molecular mobility required for growth decreases exponentially. A maximum crystallization rate therefore occurs between preferred temperatures of nucleation and growth. Cooling rate also affects nucleation, with slow cooling allowing the maintenance of a steady-state nucleation rate. Rapid cooling, on the other hand, prevents full development of nucleation and thereby facilitates glass formation (Petit \& Coquerel, 2006; Yu, 2001).

Amorphous solids offer certain pharmaceutical advantages over their crystalline counterparts. They are more soluble, have higher dissolution rates, and in some cases they may even have better compression characteristics than corresponding crystals. Therefore, we may not necessarily want amorphous APIs to recrystallize. Some pharmaceuticals have a natural tendency to exist as amorphous solids, while others require deliberate prevention of recrystallization to enter, and remain in, the amorphous state. Contemporary research into 
the stabilization of amorphous pharmaceuticals focuses on three key areas: (1) the use of additives for the stabilization of labile substances (e.g. proteins and peptides) during processing and storage; (2) prevention of crystallization of excipients that must remain amorphous to perform their intended functions; and (3) determining the appropriate storage conditions for optimal stability of amorphous materials (Yu, 2001).

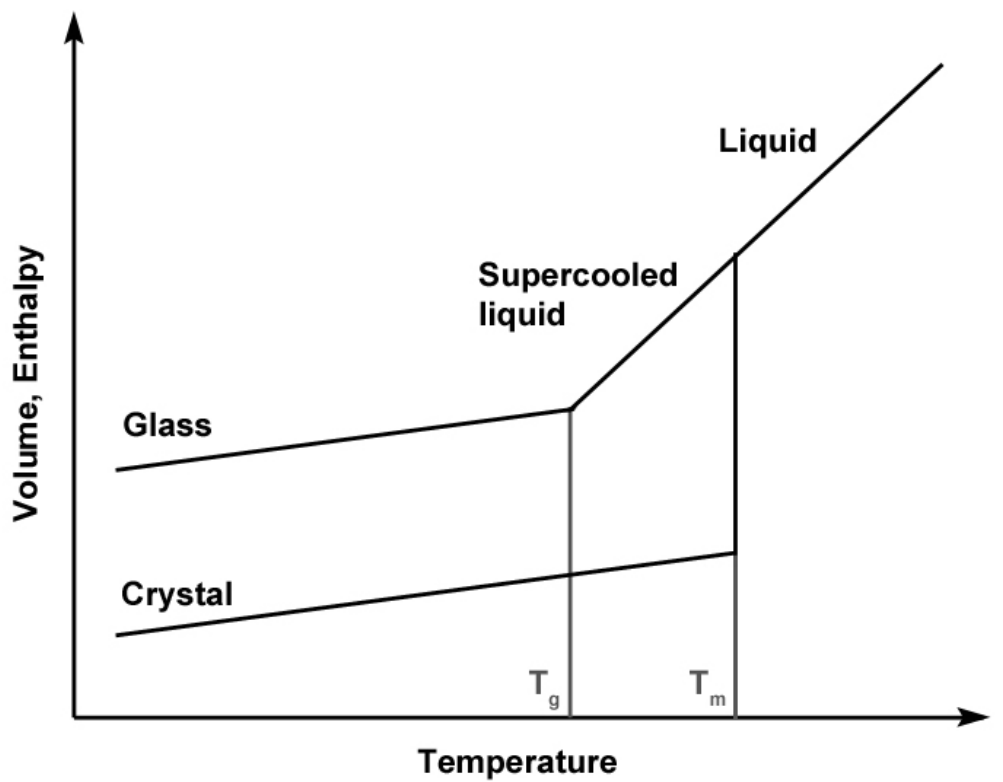

Fig. 9. Schematic representation of enthalpy or volume variations as a function of temperature for condensed materials. (Adapted, with permission of John Wiley and Sons, from Petit \& Coquerel, 2006.)

\subsection{Recrystallization through Physical Vapor Deposition}

Physical vapor deposition (PVD) is an atomistic deposition process in which material is vaporized from a solid or liquid source in the form of atoms or molecules and transported in the form of vapor through a low pressure environment to the substrate where it condenses (Mattox, 1998). During the PVD process, molecules unpack from the original crystal lattice and then recrystallize in the new lattice (Byrn et al., 1999).

The research into pharmaceutical applications of PVD has, to date, largely been limited to the production of amorphous phases - particularly ones with enhanced stability. Although we have long known that sublimation may be used for the production of polymorphs, comparatively few papers have been published on recrystallization via PVD. This is surprising when one considers that approximately two-thirds of organic compounds sublime (Guillory, 1999) and most commercially available APIs are crystalline.

Studies on the sublimation-based recrystallization of the following APIs and organic compounds have been published: anthranilic acid (Carter \& Ward, 1994); 9,10anthraquinone-2-carboxilic acid (Tsai et al., 1993); 1,1-bis(4-hydroxyphenyl)cyclohexane 
(Sarma et al., 2006); caffeine (Griesser et al., 1999; Carlton, 2011); carbamazepine (Griesser et al., 1999; Zeitler et al., 2007); 1,3-dimethyluracil (Sakiyama \& Imamura, 1989); ibuprofen (Perlovich et al., 2004;); malonamide (Sakiyama \& Imamura, 1989); nevirapine (Figure 10) (Stieger \& Liebenberg, 2009); stanozolol (Karpinska et al., 2011); and theophylline (Fokkens et al., 1983; Griesser et al., 1999).
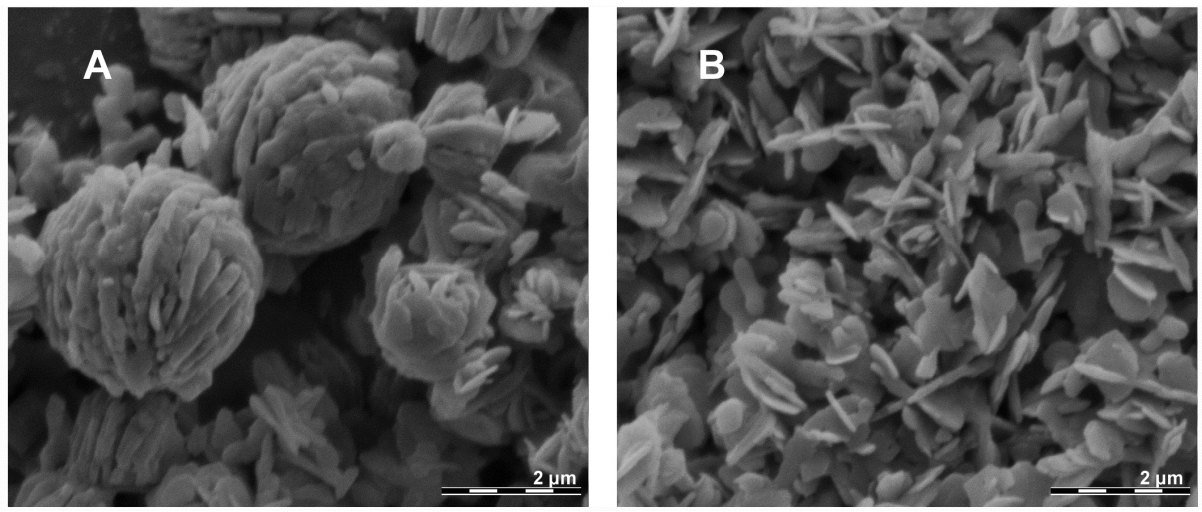

Fig. 10. Micro-spherical aggregates of nano-crystalline nevirapine prepared by PVD (A), the individual crystallites of which could be obtained through light grinding (B).

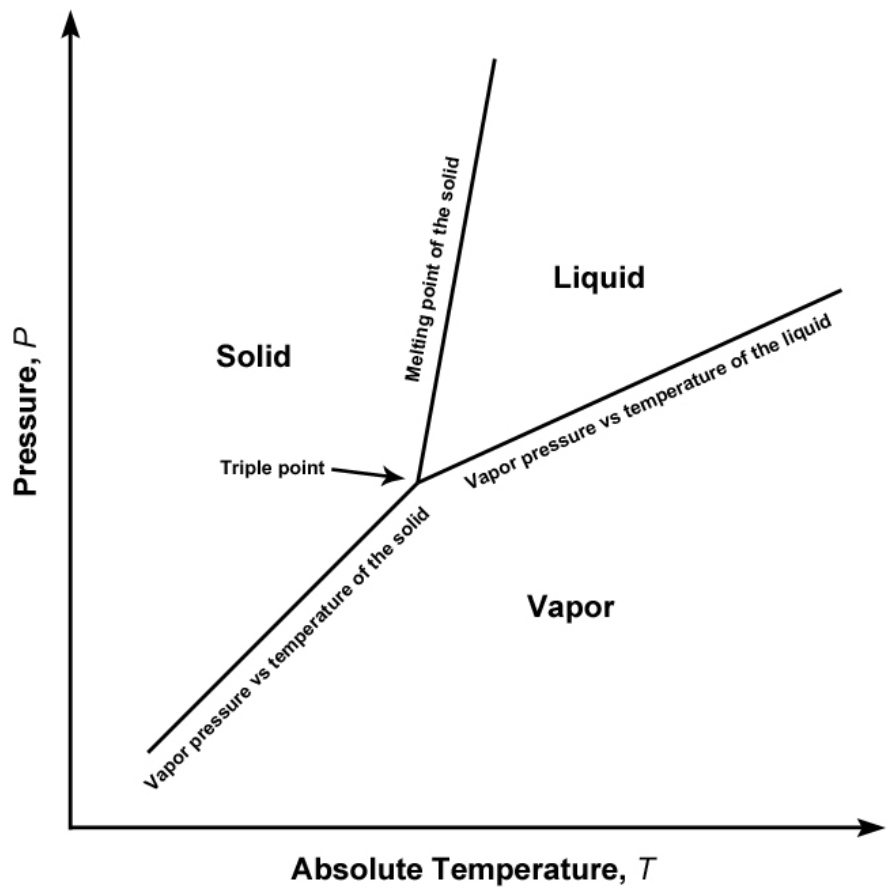

Fig. 11. Pressure-temperature diagram of a one-component crystalline solid for which only one solid phase exists. (Adapted, with permission of Dover Publications, from Ricci, 1966.) 
Figure 11 demonstrates how transformations mediated by the vapor phase are highly dependent on vapor pressure and therefore also on temperature (Byrn et al., 1999). Vapor of an API can be generated by its liquid phase once melted (evaporation) or by its solid phase (sublimation). The vast majority of pharmaceutical actives are organic compounds and, as such, they are sensitive to heat degradation. It is well-known that many APIs degrade when melted. It follows that pharmaceutical PVD processes should ideally operate at low pressure and temperature to obtain vapor through sublimation, using compounds with sufficient thermal stability.

Figure 12 illustrates how it is possible to obtain different enantiotropic polymorphs through vapor deposition. At a pressure sufficiently low for sublimation to take place the solid API becomes a vapor, at which point it is no longer Solid A or B because polymorphism is a solid-state phenomenon. The temperature at which the vapor phase recrystallizes determines which polymorph is obtained (provided the pressure is sufficiently low). It can generally be assumed that unstable/metastable polymorphs (in this example, Solid A) will form preferentially at lower temperatures and stable polymorphs (Solid B) can be expected at higher temperatures (Guillory, 1999). This is why the temperature, and distance from the solid, of the collection surface is so important in PVD systems.

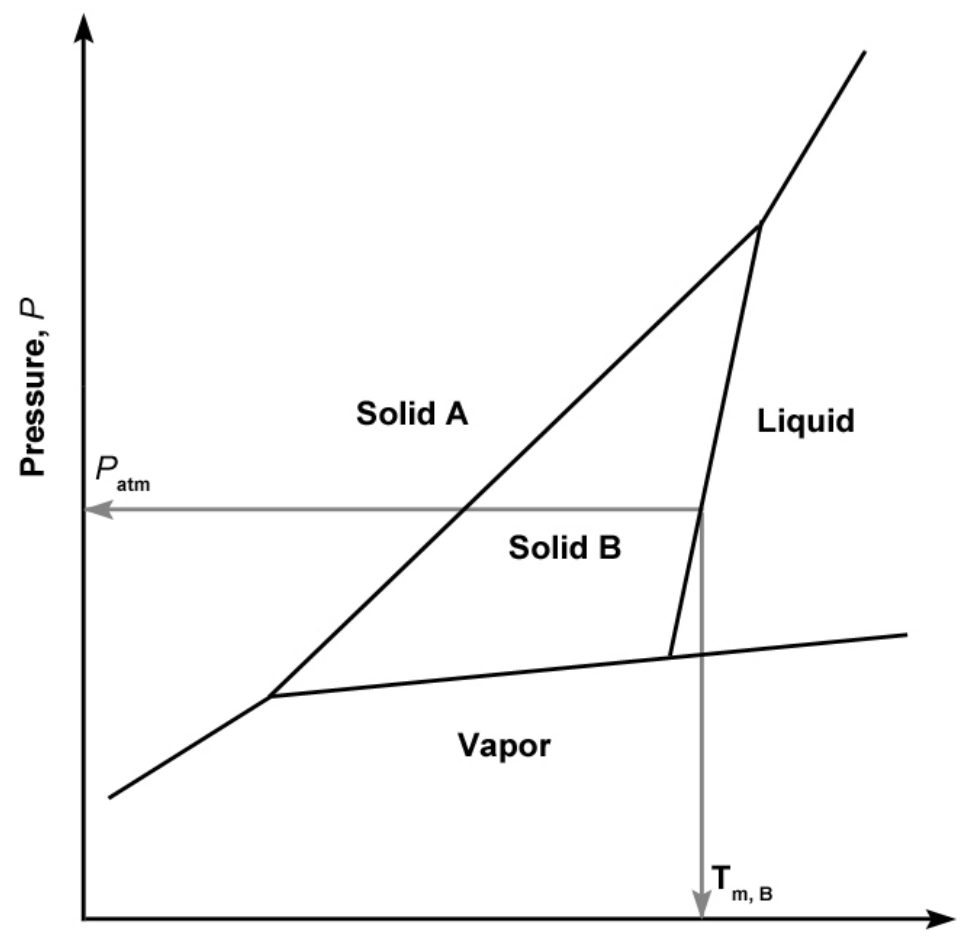

Absolute Temperature, $T$

Fig. 12. Pressure-temperature diagram of a one-component crystalline solid with enantiotropic behavior. (Adapted, with permission of John Wiley and Sons, from Lohani \& Grant, 2006.) 
It has, incidentally, been found that recrystallization from the vapor phase can also be affected or controlled by the nature of the substrate (Carter \& Ward, 1994) as has been shown for solvent recrystallization (Rodríguez-Spong et al., 2004).

\section{Conclusion}

Polymorphs of active pharmaceutical ingredients can be obtained though recrystallization processes based on solvents, solid-state transitions and vapor deposition. When the possible number of experimental variations on each of these is considered, it is easy to become overwhelmed by the task of comprehensive polymorph screening. Indeed, it is no wonder that some polymorphs go undetected for decades or longer. Various semi-automated and high-throughput methods have been developed to assist in this daunting task. The efforts of researchers laboring towards automation are, however "subverted" by colleagues who continually find novel ways of accessing hidden polymorphs! There is, to date, no single method that can identify all possible solid forms of an API.

\section{Acknowledgement}

We thank North-West University (Potchefstroom Campus) and the National Research Foundation of South Africa for providing research support.

\section{References}

Banga, S., Chawla, G. \& Bansal, A.K. 2004. New Trends in the Crystallisation of Active Pharmaceutical Ingredients. Business Briefing: Pharmagenerics, p. 1-5.

Beckman, W. 2000. Seeding the Desired Polymorph: Background, Possibilities, Limitations, and Case Studies. Organic Process Research \& Development, 4:372-383.

Bernstein, J. 2002. Polymorphism in Molecular Crystals. Oxford:Clarendon Press. 410 p.

Blagden, N., De Matas, M., Gavan, P.T. \& York, P. 2007. Crystal Engineering of Active Pharmaceutical Ingredients to Improve Solubility and Dissolution Rates. Advanced Drug Delivery Reviews, 59(7):617-630.

Boldyreva, E.V. 2003. High-Pressure-Induced Structural Changes in Molecular Crystals Preserving the Space Group Symmetry: Anisotropic Distortion/Isosymmetric Polymorphism. Crystal Engineering, 6(4):235-254.

Boistelle, R. \& Astier, J.P. 1988. Crystallization Mechanisms in Solution. Journal of Crystal Growth, 90(1-3):14-30.

Braatz, R.D. 2002. Advanced Control of Crystallization Processes. Annual Reviews in Control, 26(1):87-99.

Brittain, H.G. \& Fiese, E.F. 1999. Effects of Pharmaceutical Processing on Drug Polymorphs and Solvates. (In Brittain, H.G. Ed. Polymorphism in Pharmaceutical Solids. Volume 95: Drugs and the Pharmaceutical Sciences. New York: Marcel Dekker.) p. 331-361.

Brittain, H.G. \& Grant, D.J.W. 1999. Effects of Polymorphism and Solid-State Solvation on Solubility and Dissolution Rate. (In Brittain, H.G. Ed. Polymorphism in Pharmaceutical Solids. Volume 95: Drugs and the Pharmaceutical Sciences. New York: Marcel Dekker.) p. 279-330.

Byrn, S.R., Pfeiffer, R.R. \& Stowell, J.G. 1999. Solid-State Chemistry of Drugs. $2^{\text {nd }}$ edition. West Lafayette: SSCI. 574 p. 
Capes, J.S. \& Cameron, R.E. 2007. Effect of Polymer Addition on the Contact Line Crystallization of Paracetamol. CrystEngComm, 9:84-90.

Carlton, R.A. 2011. Pharmaceutical Microscopy. New York: Springer. 322 p.

Carter, P.W. \& Ward, M.D. 1994. Directing Polymorph Selectivity During Nucleation of Anthranilic Acid on Molecular Substrates. Journal of the American Chemical Society, 116(2):769-770.

Carstensen, J.T., Ertell, C. \& Geoffrey, J. 1993. Physico-Chemical Properties of Particulate Matter. Drug Development and Industrial Pharmacy, 19(1-2):195-219.

Doherty, C. \& York, P. 1988. Frusemide Crystal Forms: Solid State and Physicochemical Analyses. International Journal of Pharmaceutics, 47(1-3):141-155.

Douillet, J., Stevenson, N., Lee, M., Mallet, F., Ward, R., Aspin, P., Dennehy, D.R. \& Camus, L. 2011. Development of a Solvate as an Active Pharmaceutical Ingredient: Developability, Crystallisation and Isolation Challenges. Journal of Crystal Growth, article in press.

Fabbiani, F.P.A., Allan, D.R., David, W.I.F., Moggach, S.A., Parsons, S. \& Pulham, C.R. 2004. High-Pressure Recrystallization - A Route to New Polymorphs and Solvates. CrystEngComm, 6(82):504-511.

Florence, A.T. \& Attwood, D. 2006. Physicochemical Principles of Pharmacy. $4^{\text {th }}$ edition. London: Pharmaceutical Press. 492 p.

Fokkens, J.G., Van Amelsfoort, J.G.M., De Blaey, C.J., De Kruif, C.G. \& Wilting, J. 1983. A Thermodynamic Study of the Solubility of Theophylline and its Hydrate. International Journal of Pharmaceutics, 14(1):79-93.

Fujiwara, M., Nagy, Z.K., Chew, J.W. \& Braatz, R.D. 2005. First-Principles and Direct Design Approaches for the Control of Pharmaceutical Crystallization. Journal of Process Control, 15(5):493-504.

Garetz, B.A., Aber, J.E., Goddard, N.L., Young, R.G. \& Myerson, A.S. 1996. Nonphotochemical, Polarization-Dependent, Laser-Induced Nucleation in Supersaturated Aqueous Urea Solutions. Physical Review Letters, 77:3475-3476.

Grant, D.J.W. 1999. Theory and Origin of Polymorphism. (In Brittain, H.G. Ed. Polymorphism in Pharmaceutical Solids. Volume 95: Drugs and the Pharmaceutical Sciences. New York: Marcel Dekker.) p. 1-33.

Griesser, U.J. 2006. The Importance of Solvates. (In Hilfiker, R. Ed. Polymorphism in the Pharmaceutical Industry. Germany: Wiley-VCH.) p. 211-233.

Griesser, U.J., Szelagiewicz, M., Hofmeier, U.C., Pitt, C. \& Cianferani, S. 1999. Vapor Pressure and Heat of Sublimation of Crystal Polymorphs. Journal of Thermal Analysis and Calorimetry, 57(1):45-60.

Guillory, J.K. 1999. Generation of Polymorphs, Hydrates, Solvates and Amorphous Solids. (In Brittain, H.G. Ed. Polymorphism in Pharmaceutical Solids. Volume 95: Drugs and the Pharmaceutical Sciences. New York: Marcel Dekker.) p. 183-225.

Haleblian, J.K. 1975. Characterization of Habits and Crystalline Modifications of Solids and their Pharmaceutical Applications. Journal of Pharmaceutical Sciences, 64:1269-1288.

Karpinska, J., Erxleben, A. \& McArdle, P. 2011. 17 $\beta$-Hydroxy-17a-methylandrostanol[3,2c]pyrazole, Stanozolol: The Crystal Structures of Polymorphs 1 and 2 and 10 Solvates. Crystal Growth \& Design, 11(7):2829-2838.

Kim, S., Wei, C. \& Kiang, S. 2003. Crystallization Process Development of an Active Pharmaceutical Ingredient and Particle Engineering via the Use of Ultrasonics and Temperature Cycling. Organic Process Research Development, 7(6):997-1001. 
Karpinski, P.H. \& Wey, J.S. 2001. Precipitation Processes. (In Myerson, A.S. Ed. Handbook of Industrial Crystallization. 2nd edition. Woburn: Butterworth-Heinemann.) p. 141-159.

Kondepuddi, D.K., Kaufmann, R.J. \& Singh, N. 1990. Chiral Symmetry Breaking in Sodium Chlorate Crystallization. Science, 250(4983):975-976.

Kondepuddi, D.K. \& Sabanayagam, C. 1994. Secondary Nucleation that Leads to Chiral Symmetry Breaking in Stirred Crystallization. Chemical Physics Letters, 217(4):364-368.

Kuzmenko, I., Rapaport, H., Kjaer, K., Als-Nielsen, J., Weissbuch, I., Lahav, M. \& Leiserowitz, L. 2001. Design and Characterization of Crystalline Thin Film Architectures at the Air-Liquid Interface: Simplicity to Complexity. Chemical Reviews, 101(6):1659-1696.

Lipinski, C.A., Lombardo, F., Dominy, B.W. \& Feeney, P.J. 1997. Experimental and Computational Approaches to Estimate Solubility and Permeability in Drug Discovery and Development Settings. Advanced Drug Delivery Reviews, 46:3-26.

Lohani, S. \& Grant, J.W.G. 2006. Thermodynamics of Polymorphs. (In Hilfiker, R. Ed. Polymorphism in the Pharmaceutical Industry. Germany: Wiley-VCH.) p. 21-42.

Manek, R.V. \& Kolling, W.M. 2004. Influence of Moisture on the Crystal Forms of Niclosamide Obtained from Acetone and Ethyl Acetate. AAPS PharmSciTech, 5(1):101-108.

Mattox, D.M. 2010. Handbook of Physical Vapor Deposition (PVD) Processing. 2nd edition. Oxford: William Andrew. 792 p.

McBride, J.M. \& Carter, R.L. 1991. Spontaneous Resolution by Stirred Crystallization. Angewandte Chemie, 30(3):293-295.

Mullin, J.W. 2001. Crystallization. $4^{\text {th }}$ edition. Oxford: Butterworth-Heinemann. p. 322.

Ostwald, F.W. 1897. Studien uber die Bildung und Umwandlung fester Korper. Zeitschrift für Physikalische Chemie, 22:289.

Nonoyama, N., Hanaki, K. \& Yabuki, Y. 2006. Constant Supersaturation Control of Antisolvent-Addition Batch Crystallization. Organic Process Research \& Development, 10(4):727-732.

Perlovich, G.L., Kurkov, S.V., Hansen, L.K. \& Bauer-Brandl, A. 2004. Thermodynamics of Sublimation, Crystal Lattice Energies, and Crystal Structures of Racemates and Enantiomers: (+)- and (-)-Ibuprofen. Journal of Pharmaceutical Sciences, 93(3):654-666.

Petit, S. \& Coquerel, G. 2006. The Amorphous State. (In Hilfiker, R. Ed. Polymorphism in the Pharmaceutical Industry. Germany: Wiley-VCH.) p. 259-285.

Ricci, J.E. 1966. The Phase Rule and Heterogeneous Equilibrium. New York: Dover Publications. $505 \mathrm{p}$.

Rodríguez-Homedo, N., Kelly, R.C., Sinclair, B.D. \& Miller, J.M. 2006. Crystallization: General Principles and Significance on Product Development. (In Swarbrick, J. Ed. Encyclopedia of Pharmaceutical Technology. $3^{\text {rd }}$ edition. New York: Informa Healthcare.) p. 834-857.

Rodríguez-Spong, B., Price, C.P., Jayasankar, A., Matzger, A.J. \& Rodríguez-Homedo, N. 2004. General Principles of Pharmaceutical Solid Polymorphism: A Supramolecular Perspective. Advanced Drug Delivery Reviews, 56:241-274.

Rouhi, A.M. 2003. The Right Stuff. Chemical E Engineering News, 81(8):32-35.

Sakiyama, M. \& Imamura, A. 1989. Thermoanalytical Characterization of 1,3-Dimethyluracil and Malonamide Crystals. Thermochimica Acta, 142(2):365-370.

Sarma, B., Roy, S. \& Nangia, A. 2006. Polymorphs of 1,1-Bis(4-hydroxyphenyl)cyclohexane and Multiple $Z^{\prime}$ Crystal Structures by Melt and Sublimation Crystallization. Chemical Communications, 47:4918-4920. 
Shalaev, E. \& Zografi, G. 2002. The Concept of 'Structure' in Amorphous Solids from the Perspective of the Pharmaceutical Sciences. (In Levine, H. Ed. Amorphous Food and Pharmaceutical Systems. Cambridge: The Royal Society of Chemistry.) p. 11-30.

Shekunov, B.Y. \& York, P. 2000. Crystallization Processes in Pharmaceutical Technology and Drug Delivery Design. Journal of Crystal Growth, 211(1-4):122-136.

Shinde, K.R., Patil, S.D. \& Dhake, A.S. 2011. Recrystallization. Indian Streams Research Journal, $1(4)$.

Skonieczny, S. 2009. Crystallization. University of Toronto course notes for CHM249. p. 1-18.

Stieger, N., Caira, M.R., Liebenberg, W., Tiedt, L.R., Wessels, J.C. \& De Villiers, M.M. 2010a. Influence of the Composition of Water/Ethanol Mixtures on the Solubility and Recrystallization of Nevirapine. Crystal Growth \& Design, 10(9):3859-3868.

Stieger, N. \& Liebenberg, W. 2009. Method for Increasing the Solubility of a Transcriptase Inhibitor Composition. Patent application PCT/IB2010/055077 (priority date 2009-11-10).

Stieger, N., Liebenberg, W. \& Caira, M.R. 2009. Method of Producing a Polymorph Form. Patent application PCT/IB2010/055808 (priority date 2009-12-17).

Stieger, N., Liebenberg, W., Wessels, J.C., Samsodien, H. \& Caira, M.R. 2010b. Channel Inclusion of Primary Alcohols in Isostructural Solvates of the Antiretroviral Nevirapine: an X-Ray an Thermal Analysis Study. Structural Chemistry, 21(4):771-777.

Stoica, C., Verwer, P., Meekes, H., Van Hoof, P.J.C.M., Kaspersen, F.M. \& Vlieg, E. 2004. Understanding the Effect of a Solvent on the Crystal Habit. Crystal Growth \& Design, 4(4):765-768.

Threlfall, T. 2000. Crystallisation of Polymorphs: Thermodynamic Insight into the Role of Solvent. Organic Process Research \& Development, 4(5):384-390.

Tiwary, A.K. 2006. Crystal Habit Changes and Dosage Form Performance. (In Swarbrick, J. Ed. Encyclopedia of Pharmaceutical Technology. $3^{\text {rd }}$ edition. New York: Informa Healthcare.) p. 822.

Togkalidou, T., Tung, H., Sun, Y., Andrews, A. \& Braatz, R.D. 2002. Solution Concentration Prediction for Pharmaceutical Crystallization Processes Using Robust Chemometrics and ATR FTIR Spectroscopy. Organic Process Research Development, 6(3):317-322.

Tros de Ilarduya, M.C., Martin, C., Goňi, M.M. \& Martinez-Uhárriz, M.C. 1997. Dissolution Rates of Polymorphs and Two New Pseudopolymorphs of Sulindac. Drug Development and Industrial Pharmacy, 23:1095-1098.

Tsai, S., Kuo, S. \& Lin, S. 1993. Physicochemical Characterization of 9,10-anthraquinone-2carboxilic acid. Journal of Pharmaceutical Sciences, 82(12):1250-1254.

Tung, H., Paul, E.L., Midler, M. \& McCauly, J.A. 2009. Crystallization of Organic Compounds: An Industrial Perspective. New Jersey: John Wiley \& Sons. 289 p.

$\mathrm{Yu}$, L. 2001. Amorphous Pharmaceutical Solids: Preparation, Characterization and Stabilization. Advanced Drug Delivery Reviews, 48(1):27-42.

Zaccaro, J., Matic, J., Myerson, A.S. \& Garetz, B.A. 2001. Nonphotochemical, Laser-Induced Nucleation of Supersaturated Aqueous Glycine Produces Unexpected $\gamma^{-}$ Polymorph. Crystal Growth \& Design, 1(1):5-8.

Zeitler, J.A., Taday, P.F., Gordon, K.C., Pepper, M. \& Rades, T. 2007. New Insights into the Solid-State Transition Mechanism in Carbamazepine Polymorphs by TimeResolved Terahertz Spectroscopy. ChemPhysChem, 8(13):1924-1927.

Zhou, G.X., Fujiwara, M., Woo, X.Y., Rusli, E., Tung, H., Starbuck, C., Davidson, O., Ge, Z. \& Braatz, R.D. 2006. Direct Design of Pharmaceutical Antisolvent Crystallization through Concentration Control. Crystal Growth \& Design, 6(4):892-898. 
(C) 2012 The Author(s). Licensee IntechOpen. This is an open access article distributed under the terms of the Creative Commons Attribution 3.0 License, which permits unrestricted use, distribution, and reproduction in any medium, provided the original work is properly cited. 